\title{
On relative ranks of finite transformation semigroups with restricted range
}

\author{
Ilinka Dimitrova \\ Faculty of Mathematics and Natural Science \\ South-West University "Neofit Rilski" \\ 2700 Blagoevgrad, Bulgaria \\ e-mail: ilinka_dimitrova@swu.bg \\ Jörg Koppitz \\ Institute of Mathematics and Informatics \\ Bulgarian Academy of Sciences \\ 1113 Sofia, Bulgaria \\ e-mail:koppitz@math.bas.bg
}

June 16, 2020

\begin{abstract}
In this paper, we determine the relative rank of the semigroup $\mathcal{T}(X, Y)$ of all transformations on a finite chain $X$ with restricted range $Y \subseteq X$ modulo the set $\mathcal{O P}(X, Y)$ of all orientation-preserving transformation in $\mathcal{T}(X, Y)$. Moreover, we state the relative rank of the semigroup $\mathcal{O P}(X, Y)$ modulo the set $\mathcal{O}(X, Y)$ of all order-preserving transformations in $\mathcal{O P}(X, Y)$. In both cases we characterize the minimal relative generating sets.
\end{abstract}

Key words: transformation semigroups with restricted range, orderpreserving transformations, orientation-preserving transformations, relative rank, relative generating sets.

2010 Mathematics Subject Classification: 20M20

\section{Introduction and Preliminaries}

Let $S$ be a semigroup. The rank of $S($ denoted by $\operatorname{rank} S)$ is defined to be the minimal number of elements of a generating set of $S$. The ranks of 
various well known semigroups have been calculated [7, 8, 10, 11. For a set $A \subseteq S$, the relative rank of $S$ modulo $A$, denoted by $\operatorname{rank}(S: A)$, is the minimal cardinality of a set $B \subseteq S$ such that $A \cup B$ generates $S$. It follows immediately from the definition that $\operatorname{rank}(S: \emptyset)=\operatorname{rank} S, \operatorname{rank}(S: S)=0$, $\operatorname{rank}(S: A)=\operatorname{rank}(S:\langle A\rangle)$ and $\operatorname{rank}(S: A)=0$ if and only if $A$ is a generating set for $S$. The relative rank of a semigroup modulo a suitable set was first introduced by Ruškuc in [14] in order to describe the generating sets of semigroups with infinite rank. In [12], Howie, Ruškuc, and Higgins considered the relative ranks of the monoid $\mathcal{T}(X)$ of all full transformations on $X$, where $X$ is an infinite set, modulo some distinguished subsets of $\mathcal{T}(X)$. They showed that $\operatorname{rank}(\mathcal{T}(X): \mathcal{S}(X))=2, \operatorname{rank}(\mathcal{T}(X): \mathcal{E}(X))=2$ and $\operatorname{rank}(\mathcal{T}(X): J)=0$, where $\mathcal{S}(X)$ is the symmetric group on $X, \mathcal{E}(X)$ is the set of all idempotent transformations on $X$ and $J$ is the top $\mathcal{J}$-class of $\mathcal{T}(X)$, i.e. $J=\{\alpha \in \mathcal{T}(X):|X \alpha|=|X|\}$. But also if the rank is finite, the relative rank gives information about the generating sets. In the present paper, we will determine the relative rank for a particular semigroup of transformations on a finite set.

Let $X$ be a finite chain, say $X=\{1<2<\cdots<n\}$, for a natural number $n$. A transformation $\alpha \in \mathcal{T}(X)$ is called order-preserving if $x \leq y$ implies $x \alpha \leq y \alpha$, for all $x, y \in X$. We denote by $\mathcal{O}(X)$ the submonoid of $\mathcal{T}(X)$ of all order-preserving full transformations on $X$. The relative rank of $\mathcal{T}(X)$ modulo $\mathcal{O}(X)$ was considered by Higgins, Mitchell, and Ruškuc in 9]. They showed that $\operatorname{rank}(\mathcal{T}(X): \mathcal{O}(X))=1$, when $X$ is an arbitrary countable chain or an arbitrary well-ordered set, while $\operatorname{rank}(\mathcal{T}(\mathbb{R}): \mathcal{O}(\mathbb{R}))$ is uncountable, by considering the usual order of the set $\mathbb{R}$ of real numbers. In 2], Dimitrova, Fernandes, and Koppitz studied the relative rank of the semigroup $\mathcal{O}(X)$ modulo $J=\{\alpha \in \mathcal{O}(X):|X \alpha|=|X|\}$, for an infinite countable chain $X$. We say that a transformation $\alpha \in \mathcal{T}(X)$ is orientationpreserving if there are subsets $X_{1}, X_{2} \subseteq X$ with $\emptyset \neq X_{1}<X_{2}$, (i.e. $x_{1}<x_{2}$ for $x_{1} \in X_{1}$ and $\left.x_{2} \in X_{2}\right), X=X_{1} \cup X_{2}$, and $x \alpha \leq y \alpha$, whenever either $(x, y) \in X_{1}^{2} \cup X_{2}^{2}$ with $x \leq y$ or $(x, y) \in X_{2} \times X_{1}$. Note that $X_{2}=\emptyset$ provides $\alpha \in \mathcal{O}(X)$. We denote by $\mathcal{O P}(X)$ the submonoid of $\mathcal{T}(X)$ of all orientation-preserving full transformations on $X$. An equivalent notion of an orientation-preserving transformation was first introduced by McAlister in 13 and, independently, by Catarino and Higgins in [1]. It is clear that $\mathcal{O}(X)$ is a submonoid of $\mathcal{O P}(X)$, i.e. $\mathcal{O}(X) \subset \mathcal{O P}(X) \subset \mathcal{T}(X)$. It is interesting to note that the relative rank of $\mathcal{T}(X)$ modulo $\mathcal{O P}(X)$ as well as the relative rank of $\mathcal{O P}(X)$ modulo $\mathcal{O}(X)$ is one (see [1, 12]), but the situation will change if one considers transformations with restricted range.

Let $Y=\left\{a_{1}<a_{2}<\cdots<a_{m}\right\}$ be a nonempty subset of $X$, for a 
natural number $m \leq n$, and denote by $\mathcal{T}(X, Y)$ the subsemigroup $\{\alpha \in$ $\mathcal{T}(X): X \alpha \subseteq Y\}$ of $\mathcal{T}(X)$ of all transformations with range (image) restricted to $Y$. The set $\mathcal{T}(X, Y)$ coincides with $\mathcal{T}(X)$, whenever $Y=X$ (i.e. $m=n$ ). In 1975, Symons [15] introduced and studied the semigroup $\mathcal{T}(X, Y)$, which is called semigroup of transformations with restricted range. Recently, the rank of $\mathcal{T}(X, Y)$ was computed by Fernandes and Sanwong in [6]. They proved that the rank of $\mathcal{T}(X, Y)$ is the Sterling number $S(n, m)$ of second kind with $|X|=n$ and $|Y|=m$. The rank of the order-preserving counterpart $\mathcal{O}(X, Y)$ of $\mathcal{T}(X, Y)$ was studied in [4] by Fernandes, Honyam, Quinteiro, and Singha. The authors found that $\operatorname{rank} \mathcal{O}(X, Y)=\left(\begin{array}{c}n-1 \\ m-1\end{array}\right)+\left|Y^{\#}\right|$, where $Y^{\#}$ denotes the set of all $y \in Y$ with one of the following properties: (i) $y$ has no successor in $X$; (ii) $y$ is no successor of any element in $X$; (iii) both the successor of $Y$ and the element whose successor is $y$ belong to $Y$. Moreover, the regularity and the rank of the semigroup $\mathcal{O P}(X, Y)$ were studied by the same authors in [5]. They showed that $\operatorname{rank} \mathcal{O P}(X, Y)=\left(\begin{array}{c}n \\ m\end{array}\right)$. In [16], Tinpun and Koppitz studied the relative rank of $\mathcal{T}(X, Y)$ modulo $\mathcal{O}(X, Y)$ and proved that $\operatorname{rank}(\mathcal{T}(X, Y): \mathcal{O}(X, Y))=S(n, m)-\left(\begin{array}{c}n-1 \\ m-1\end{array}\right)+a$, where $a \in\{0,1\}$ depending on the set $Y$. In this paper, we determine the relative rank of $\mathcal{O P}(X, Y)$ modulo $\mathcal{O}(X, Y)$ as well as the relative rank of $\mathcal{T}(X, Y)$ modulo $\mathcal{O P}(X, Y)$.

Let $\alpha \in \mathcal{T}(X, Y)$. The kernel of $\alpha$ is the equivalence relation ker $\alpha$ with $(x, y) \in \operatorname{ker} \alpha$ if $x \alpha=y \alpha$. It corresponds uniquely to a partition on $X$. This justifies to regard $\operatorname{ker} \alpha$ as a partition on $X$. We will call a block of this partition as ker $\alpha$-class. In particular, the sets $x \alpha^{-1}=\{y \in X: y \alpha=x\}$, for $x \in X \alpha$, are the ker $\alpha$-classes. We say that a partition $P$ is a subpartition of a partition $Q$ of $X$ if for all $p \in P$ there is $q \in Q$ with $p \subseteq q$. A set $T \subseteq X$ with $\left|T \cap x \alpha^{-1}\right|=1$, for all $x \in X \alpha$, is called a transversal of $\operatorname{ker} \alpha$. Let $A \subseteq X$. Then $\left.\alpha\right|_{A}: A \rightarrow Y$ denotes the restriction of $\alpha$ to $A$ and $A$ will be called convex if $x<y<z$ with $x, z \in A$ implies $y \in A$.

Let $l \in\{1, \ldots, m\}$. We denote by $\mathcal{P}_{l}$ the set of all partitions $\left\{A_{1}, \ldots, A_{l}\right\}$ of $X$ such that $A_{2}<A_{3}<\cdots<A_{l}$ are convex sets (if $l>1$ ) and $A_{1}$ is the union of two convex sets with $1, n \in A_{1}$. Further, let $\mathcal{Q}_{l}$ be the set of all partitions $\left\{A_{1}, \ldots, A_{l}\right\}$ of $X$ such that $A_{1}<A_{2}<\cdots<A_{l}$ are convex and let $\mathcal{R}_{l}$ be the set of all partitions of $X$, which not belong to $\mathcal{Q}_{l} \cup \mathcal{P}_{l}$. We observe that $\operatorname{ker} \beta \in \mathcal{Q}_{l} \cup \mathcal{P}_{l}$, whenever $\beta \in \mathcal{O P}(X, Y)$ with $|X \beta|=l$. In particular, $\operatorname{ker} \beta \in \mathcal{Q}_{l}$, whenever $\beta \in \mathcal{O}(X, Y)$. 
Let us consider the case $l=m>1$. For $P \in \mathcal{P}_{m}$ with the blocks $A_{1}, A_{2}<\cdots<A_{m}$, let $\alpha_{P}$ be the transformation on $X$ defined by

$$
x \alpha_{P}:=a_{i} \text {, whenever } x \in A_{i} \text { for } 1 \leq i \leq m
$$

in the case $1 \notin Y$ or $n \notin Y$ and

$$
x \alpha_{P}:= \begin{cases}a_{i+1}, & \text { if } x \in A_{i} \text { for } 1 \leq i<m \\ a_{1} & \text { if } x \in A_{m}\end{cases}
$$

in the case $1, n \in Y$. Clearly, $\operatorname{ker} \alpha_{P}=P$. With $X_{1}=\left\{1, \ldots, \max A_{m}\right\}$, $X_{2}=\left\{\max A_{m}+1, \ldots, n\right\}$ in the case $1 \notin Y$ or $n \notin Y$ and $X_{1}=\left\{1, \ldots, \max A_{m-1}\right\}$, $X_{2}=\left\{\max A_{m-1}+1, \ldots, n\right\}$ in the case $1, n \in Y$, where $\max A_{m}\left(\max A_{m-1}\right)$ denotes the greatest element in the set $A_{m}\left(A_{m-1}\right.$, respectively), we can easy verify that $\alpha_{P}$ is orientation-preserving.

Further, let $\eta \in \mathcal{T}(X, Y)$ be defined by

$x \eta:=\left\{\begin{array}{ll}a_{i+1} & \text { if } a_{i} \leq x<a_{i+1} \text { for } 1 \leq i<m \\ a_{1} & \text { if } x=a_{m} \\ a_{\Gamma} & \text { otherwise }\end{array} \quad\right.$ with $\Gamma:= \begin{cases}1 & \text { if } 1 \notin Y \\ 2 & \text { otherwise }\end{cases}$

in the case $1 \notin Y$ or $n \notin Y$ and

$$
x \eta:=\left\{\begin{array}{lll}
a_{i+1} & \text { if } \quad a_{i} \leq x<a_{i+1}, \quad 1 \leq i<m \\
a_{1}=1 & \text { if } \quad x=a_{m}=n
\end{array}\right.
$$

in the case that $1, n \in Y$. Notice that $P_{0}:=\operatorname{ker} \eta \in \mathcal{P}_{m}$ if $1 \notin Y$ or $n \notin Y$ and ker $\eta \in \mathcal{Q}_{m}$ if $1, n \in Y$. In fact, $\eta \in \mathcal{O P}(X, Y)$ with $X_{1}=\left\{1,2, \ldots, a_{m}-1\right\}$ and $X_{2}=\left\{a_{m}, a_{m}+1, \ldots, n\right\}$. Moreover, $\left.\eta\right|_{Y}$ is a permutation on $Y$, namely

$$
\left.\eta\right|_{Y}=\left(\begin{array}{cccc}
a_{1} & \ldots & a_{m-1} & a_{m} \\
a_{2} & \cdots & a_{m} & a_{1}
\end{array}\right)
$$

We will denote by $\mathcal{S}(Y)$ the set of all permutations on $Y$. Note, $\beta \in \mathcal{O}(X, Y)$ implies that either $\left.\beta\right|_{Y}$ is the identity mapping on $Y$ or $\left.\beta\right|_{Y} \notin \mathcal{S}(Y)$.

\section{The relative rank of $\mathcal{O P}(X, Y)$ modulo $\mathcal{O}(X, Y)$}

In this section, we determine the relative rank of $\mathcal{O P}(X, Y)$ modulo $\mathcal{O}(X, Y)$. A part of these results were presented at the 47-th Spring Conference of the Union of Bulgarian Mathematicians in March 2018 and are published in the proceedings of this conference [3]. 
If $m=1$ then $\mathcal{O P}(X, Y)$ is the set of all constant mappings and coincides with $\mathcal{O}(X, Y)$, i.e. $\operatorname{rank}(\mathcal{O P}(X, Y): \mathcal{O}(X, Y))=0$. So, we admit that $m>1$.

First, we will show that

$$
\mathcal{A}:=\left\{\alpha_{P}: P \in \mathcal{P}_{m}\right\} \cup\{\eta\}
$$

is a relative generating set of $\mathcal{O P}(X, Y)$ modulo $\mathcal{O}(X, Y)$. Notice that $\eta=$ $\alpha_{P_{0}}$ if $1 \notin Y$ or $n \notin Y$.

Lemma 1 For each $\alpha \in \mathcal{O P}(X, Y)$ with rank $\alpha=m$, there is $\widehat{\alpha} \in\left\{\alpha_{P}\right.$ : $\left.P \in \mathcal{P}_{m}\right\} \cup \mathcal{O}(X, Y)$ with $\operatorname{ker} \alpha=\operatorname{ker} \widehat{\alpha}$.

Proof. Let $\alpha \in \mathcal{O P}(X, Y)$ and let $X_{1}, X_{2} \subseteq X$ as in the definition of an orientation-preserving transformation. If $X_{2}=\emptyset$ then $\alpha \in \mathcal{O}(X, Y)$. Suppose now that $X_{2} \neq \emptyset$ and let $X_{1} \alpha=\left\{x_{1}<\cdots<x_{r}\right\}$ and $X_{2} \alpha=\left\{y_{1}<\right.$ $\left.\cdots<y_{s}\right\}$ for suitable natural numbers $r$ and $s$. We observe that $X_{1} \alpha$ and $X_{2} \alpha$ have at most one joint element (only $x_{1}=y_{s}$ could be possible). If $x_{1} \neq y_{s}$ then $\operatorname{ker} \alpha=\left\{x_{1} \alpha^{-1}<\cdots<x_{r} \alpha^{-1}<y_{1} \alpha^{-1}<\cdots<y_{s} \alpha^{-1}\right\}=$ ker $\widehat{\alpha}$ with $\widehat{\alpha}=\left(\begin{array}{cccccc}x_{1} \alpha^{-1} & \cdots & x_{r} \alpha^{-1} & y_{1} \alpha^{-1} & \cdots & y_{s} \alpha^{-1} \\ a_{1} & \cdots & a_{r} & a_{r+1} & \cdots & a_{r+s}\end{array}\right) \in \mathcal{O}(X, Y)$. If $x_{1}=y_{s}$ then $1, n \in x_{1} \alpha^{-1}=y_{s} \alpha^{-1}$ and $\operatorname{ker} \alpha=\operatorname{ker} \alpha_{P}$ with $P=$ $\left\{x_{1} \alpha^{-1}, x_{2} \alpha^{-1}<\cdots<x_{r} \alpha^{-1}<y_{1} \alpha^{-1}<\cdots<y_{s-1} \alpha^{-1}\right\} \in \mathcal{P}_{m}$.

Proposition $2 \mathcal{O P}(X, Y)=\langle\mathcal{O}(X, Y), \mathcal{A}\rangle$.

Proof. Let $\beta \in \mathcal{O P}(X, Y)$ with $\operatorname{rank} \beta=m$. Then there is $\theta \in\left\{\alpha_{P}\right.$ : $\left.P \in \mathcal{P}_{m}\right\} \cup \mathcal{O}(X, Y)$ with $\operatorname{ker} \beta=\operatorname{ker} \theta$ by Lemma 1. In particular, there is $r \in\{0, \ldots, m-1\}$ with $a_{1} \theta^{-1}=a_{r+1} \beta^{-1}$. Then it is easy to verify that $\beta=\theta \eta^{r}$, where $\eta^{0}=\eta^{m}$.

Admit now that $i=\operatorname{rank} \beta<m$. Suppose that $\operatorname{ker} \beta \in \mathcal{P}_{i}$, say $\operatorname{ker} \beta=$ $\left\{A_{1}, A_{2}<\cdots<A_{i}\right\}$ with $1, n \in A_{1}$. Then there is a subpartition $P^{\prime} \in \mathcal{P}_{m}$ of $\operatorname{ker} \beta$. We put $\theta=\alpha_{P^{\prime}}, a=\min X \beta$, and let $T$ be a transversal of $\operatorname{ker} \theta$. In particular, we have $Y=\left\{x\left(\left.\theta\right|_{T}\right) \eta^{k}: x \in T\right\}$ for all $k \in\{1, \ldots, m\}$. Since both mappings $\left.\theta\right|_{T}: T \rightarrow Y$ and $\left.\eta\right|_{Y}: Y \rightarrow Y$ are bijections, there is $k \in$ $\{1, \ldots, m\}$ with $a_{1}\left(\left(\left.\theta\right|_{T}\right) \eta^{k}\right)^{-1} \beta=a$ and $a_{1}\left(\left(\left.\theta\right|_{T}\right) \eta^{k+1}\right)^{-1} \beta \neq a$. Moreover, since $\left(\left.\theta\right|_{T}\right) \eta^{k}$ is a bijection from $T$ to $Y$ and both transformations $\theta \eta^{k}$ and $\beta$ are orientation-preserving, it is easy to verify that $f^{*}=\left(\left(\left.\theta\right|_{T}\right) \eta^{k}\right)^{-1} \beta$ can be extended to an orientation-preserving transformation $f$ defined by

$$
x f=\left\{\begin{array}{lll}
a_{1} f^{*} & \text { if } \quad x<a_{1} \\
a_{i} f^{*} & \text { if } \quad a_{i} \leq x<a_{i+1}, \quad 1 \leq i<m \\
a_{m} f^{*} & \text { if } \quad a_{m} \leq x
\end{array}\right.
$$


i.e. $f$ and $f^{*}$ coincide on $Y$. Moreover, $a_{1} f=a_{1} f^{*}=a_{1}\left(\left(\left.\theta\right|_{T}\right) \eta^{k}\right)^{-1} \beta=a$. In order to show that $f$ is order-preserving, it left to verify that $n f \neq a$. Assume that $n f=a$, where $n \geq a_{m}$. Then $n f=a_{m} f^{*}=a_{m} f$, i.e. $\left(n, a_{m}\right) \in \operatorname{ker} f$ and $n \eta=a_{m} \eta=a_{1}$. So, there is $x^{*} \in T$ such that $x^{*}\left(\left(\left.\theta\right|_{T}\right) \eta^{k}\right)=a_{m}$, i.e. $x^{*}=a_{m}\left(\left(\left.\theta\right|_{T}\right) \eta^{k}\right)^{-1}$. Now, we have $a=n f=a_{m} f^{*}=$ $a_{m}\left(\left(\left.\theta\right|_{T}\right) \eta^{k}\right)^{-1} \beta=a_{m}\left(\left.\eta^{k}\right|_{Y}\right)^{-1}\left(\left.\theta\right|_{T}\right)^{-1} \beta=a_{1}\left(\left.\eta\right|_{Y}\right)^{-1}\left(\left.\eta^{k}\right|_{Y}\right)^{-1}\left(\left.\theta\right|_{T}\right)^{-1} \beta=$ $a_{1}\left(\left(\left.\theta\right|_{T}\right) \eta^{k+1}\right)^{-1} \beta \neq a$, a contradiction.

Finally, we will verify that $\beta=\theta \eta^{k} f \in\langle\mathcal{O}(X, Y), \mathcal{A}\rangle$. For this let $x \in X$. Then there is $\widetilde{x} \in T$ such that $(x, \widetilde{x}) \in \operatorname{ker} \beta$. So, we have $x \theta \eta^{k} f=x \theta \eta^{k} f^{*}=$ $\widetilde{x} \theta \eta^{k}\left(\left(\left.\theta\right|_{T}\right) \eta^{k}\right)^{-1} \beta=\widetilde{x} \beta=x \beta$.

Suppose now that $\operatorname{ker} \beta \notin \mathcal{P}_{i}$ and thus, $\operatorname{ker} \beta \in \mathcal{Q}_{i}$. Let $X \beta=\left\{b_{1}, \ldots b_{i}\right\}$ such that $b_{1} \beta^{-1}<\cdots<b_{i} \beta^{-1}$. Then we define a transformation $\varphi$ by $x \varphi=a_{j}$ for all $x \in b_{j-1} \beta^{-1}$ and $2 \leq j \leq i+1$. Clearly, $\varphi \in \mathcal{O}(X, Y)$. Further, we define a transformation $\nu \in \mathcal{T}(X, Y)$ by

$$
x \nu= \begin{cases}b_{j-1} & \text { if } a_{j} \leq x<a_{j+1}, \quad 2 \leq j \leq i \\ b_{i} & \text { otherwise }\end{cases}
$$

Since $\beta$ is orientation-preserving, there is $k \in\{1, \ldots, i\}$ such that $k=i$ or $b_{1}<\cdots<b_{k-1}>b_{k}<\cdots<b_{i}$. Then $X_{1}=\left\{a_{1}, \ldots, a_{k+1}-1\right\}$ and $X_{2}=\left\{a_{k+1}, \ldots, n\right\}$ gives a partition of $X$ providing that $\nu$ is orientationpreserving. Clearly, $\operatorname{rank} \nu=i$ and $1 \nu=n \nu=b_{i}$. Thus, it is easy to verify that $\operatorname{ker} \nu \in \mathcal{P}_{i}$. Hence, $\nu \in\langle\mathcal{O}(X, Y), \mathcal{A}\rangle$ by the previous case and it remains to show that $\beta=\varphi \nu \in\langle\mathcal{O}(X, Y), \mathcal{A}\rangle$. For this let $x \in X$. Then $x \in b_{j} \beta^{-1}$ for some $j \in\{1, \ldots, i\}$, i.e. $x \varphi \nu=a_{j+1} \nu=b_{j}=x \beta$.

The previous proposition shows that $\mathcal{A}$ is a relative generating set for $\mathcal{O P}(X, Y)$ modulo $\mathcal{O}(X, Y)$. It remains to show that $\mathcal{A}$ is of minimal size.

Lemma 3 Let $B \subseteq \mathcal{O P}(X, Y)$ be a relative generating set of $\mathcal{O P}(X, Y)$ modulo $\mathcal{O}(X, Y)$. Then $\mathcal{P}_{m} \subseteq\{\operatorname{ker} \alpha: \alpha \in B\}$.

Proof. Let $P \in \mathcal{P}_{m}$. Since $\alpha_{P} \in \mathcal{O P}(X, Y)=\langle\mathcal{O}(X, Y), B\rangle$, there are $\theta_{1} \in \mathcal{O}(X, Y) \cup B$ and $\theta_{2} \in \mathcal{O P}(X, Y)$ with $\alpha_{P}=\theta_{1} \theta_{2}$. Because of $\operatorname{rank} \alpha_{P}=m$, we obtain $\operatorname{ker} \alpha_{P}=\operatorname{ker} \theta_{1}$. Since $1 \alpha_{P}=n \alpha_{P}$, we conclude that $\theta_{1} \notin \mathcal{O}(X, Y)$, i.e. $\theta_{1} \in B$ with $\operatorname{ker} \theta_{1}=\operatorname{ker} \alpha_{P}=P$.

In order to find a formula for the number of elements in $\mathcal{P}_{m}$, we have to compute the number of possible partitions of $X$ into $m+1$ convex sets. This number is $\left(\begin{array}{c}n-1 \\ m\end{array}\right)$. Thus, we have 
Remark $4\left|\mathcal{P}_{m}\right|=\left(\begin{array}{c}n-1 \\ m\end{array}\right)$.

Now, we are able to state the main result of the section. The relative rank of $\mathcal{O P}(X, Y)$ modulo $\mathcal{O}(X, Y)$ depends of the fact whether both 1 and $n$ belong to $Y$ or not.

Theorem 5 For each $1<m<n \in \mathbb{N}$,

1. $\operatorname{rank}(\mathcal{O P}(X, Y): \mathcal{O}(X, Y))=\left(\begin{array}{c}n-1 \\ m\end{array}\right)$ if $1 \notin Y$ or $n \notin Y ;$

2. $\operatorname{rank}(\mathcal{O P}(X, Y): \mathcal{O}(X, Y))=1+\left(\begin{array}{c}n-1 \\ m\end{array}\right)$ if $\{1, n\} \subseteq Y$.

Proof. 1. Note that ker $\eta \in \mathcal{P}_{m}$ and $\eta=\alpha_{P_{0}}$. Hence, the set $\mathcal{A}=\left\{\alpha_{P}\right.$ : $\left.P \in \mathcal{P}_{m}\right\}$ is a generating set of $\mathcal{O P}(X, Y)$ modulo $\mathcal{O}(X, Y)$ by Proposition 2, i.e. the relative rank of $\mathcal{O P}(X, Y)$ modulo $\mathcal{O}(X, Y)$ is bounded by the cardinality of $\mathcal{P}_{m}$, which is $\left(\begin{array}{c}n-1 \\ m\end{array}\right)$ by Remark 4 , But this number cannot be reduced by Lemma 3 .

2. Let $B \subseteq \mathcal{O P}(X, Y)$ be a relative generating set of $\mathcal{O P}(X, Y)$ modulo $\mathcal{O}(X, Y)$. By Lemma 3, we know that $\mathcal{P}_{m} \subseteq\{\operatorname{ker} \alpha: \alpha \in B\}$. Assume that the equality holds. Note that $\operatorname{ker} \eta \in \mathcal{Q}_{m}$ and $\eta$ is not order-preserving. Hence, there are $\theta_{1}, \ldots, \theta_{l} \in \mathcal{O}(X, Y) \cup B$, for a suitable natural number $l$, such that $\eta=\theta_{1} \cdots \theta_{l}$. From $\operatorname{rank} \eta=m$, we obtain $\operatorname{ker} \theta_{1}=\operatorname{ker} \eta$ and $\operatorname{rank} \theta_{i}=m$ for $i \in\{1, \ldots, l\}$ and thus, $\{1, n\} \subseteq Y$ implies $(1, n) \notin \operatorname{ker} \theta_{i}$ for $i \in\{2, \ldots, l\}$. This implies $\theta_{2}, \ldots, \theta_{l} \in \mathcal{O}(X, Y)$. Since $\operatorname{ker} \theta_{1}=\operatorname{ker} \eta \notin \mathcal{P}_{m}$, we get $\theta_{1} \in \mathcal{O}(X, Y)$, and consequently, $\eta=\theta_{1} \theta_{2} \cdots \theta_{l} \in \mathcal{O}(X, Y)$, a contradiction. So, we have verified that $\left|\mathcal{P}_{m}\right|<|B|$, i.e. the relative rank of $\mathcal{O P}(X, Y)$ modulo $\mathcal{O}(X, Y)$ is greater than $\left(\begin{array}{c}n-1 \\ m\end{array}\right)$. But it is bounded by $1+\left(\begin{array}{c}n-1 \\ m\end{array}\right)$ due to Proposition 2, This proves the assertion.

We finish this section with the characterization of the minimal relative generating sets of $\mathcal{O P}(X, Y)$ modulo $\mathcal{O}(X, Y)$. We will recognize that among them there are sets with size greater than $\operatorname{rank}(\mathcal{O P}(X, Y): \mathcal{O}(X, Y))$.

Theorem 6 Let $B \subseteq \mathcal{O P}(X, Y)$. Then $B$ is a minimal relative generating set of $\mathcal{O P}(X, Y)$ modulo $\mathcal{O}(X, Y)$ if and only if for the set $\widetilde{B}=\{\beta \in B$ : 
$\left.\operatorname{ker} \beta \in \mathcal{Q}_{m}\right\} \subseteq B$ the following three statements are satisfied:

(i) $\mathcal{P}_{m} \subseteq\{\operatorname{ker} \beta: \beta \in B \backslash \widetilde{B}\}$,

(ii) $|B \backslash \widetilde{B}|=\left|\mathcal{P}_{m}\right|$,

(iii) $\left.\eta\right|_{Y} \in\left\langle\left.\beta\right|_{Y}: \beta \in B\right\rangle$ but $\left.\eta\right|_{Y} \notin\left\langle\left.\beta\right|_{Y}: \beta \in B \backslash\{\gamma\}\right\rangle$ for any $\gamma \in \widetilde{B}$.

Proof. Suppose that the conditions $(i)-($ iii $)$ are satisfied for $\widetilde{B}=\{\beta \in$ $\left.B: \operatorname{ker} \beta \in \mathcal{Q}_{m}\right\}$. We will show that $\mathcal{A} \subseteq\langle\mathcal{O}(X, Y), B\rangle$. Let $\alpha \in \mathcal{A} \backslash\{\eta\}$. Then there is a partition $P=\left\{A_{1}, A_{2}<\cdots<A_{m}\right\} \in \mathcal{P}_{m}$ such that

$$
\alpha=\alpha_{P}=\left(\begin{array}{cccc}
A_{1} & A_{2} & \cdots & A_{m} \\
a_{1} & a_{2} & \cdots & a_{m}
\end{array}\right), \text { if } 1 \notin Y \text { or } n \notin Y,
$$

or

$$
\alpha=\alpha_{P}=\left(\begin{array}{ccccc}
A_{1} & A_{2} & \cdots & A_{m-1} & A_{m} \\
a_{2} & a_{3} & \cdots & a_{m} & a_{1}
\end{array}\right), \text { if } 1, n \in Y .
$$

Notice that in the latter case $a_{1}=1$ and $a_{m}=n$.

Further, from $(i)$ it follows that there is $\beta \in B$ with $\operatorname{ker} \beta=\operatorname{ker} \alpha_{P}$, i.e. $\beta=\alpha_{P}$ or

$$
\beta=\left(\begin{array}{ccccccc}
A_{1} & A_{2} & \cdots & A_{m-i+1} & A_{m-i+2} & \cdots & A_{m} \\
a_{i} & a_{i+1} & \cdots & a_{m} & a_{1} & \cdots & a_{i-1}
\end{array}\right)
$$

for some $i \in\{3, \ldots, m\}$. It is easy to verify that $\alpha_{P}=\beta^{k} \in\langle B\rangle$, for a suitable natural number $k$. Hence, $\left\{\alpha_{P}: P \in \mathcal{P}_{m}\right\} \subseteq\langle\mathcal{O}(X, Y), B\rangle$. Further, ker $\eta \in \mathcal{P}_{m}$, whenever $1 \notin Y$ or $n \notin Y$, and ker $\eta \in \mathcal{Q}_{m}$ otherwise. Thus, there is $\delta \in\langle\mathcal{O}(X, Y), B\rangle$ with $\operatorname{ker} \delta=\operatorname{ker} \eta$. Then we obtain as above that $\eta=\delta^{l} \in\langle\mathcal{O}(X, Y), B\rangle$, for a suitable natural number $l$. Consequently, $\langle\mathcal{O}(X, Y), \mathcal{A}\rangle \subseteq\langle\mathcal{O}(X, Y), B\rangle$. By Proposition 2, we obtain $\mathcal{O P}(X, Y)=$ $\langle\mathcal{O}(X, Y), B\rangle$. The generating set $B$ is minimal by properties $(i)$ and $(i i)$ together with Lemma 3 and by the property $(i i i)$ of $\widetilde{B}$.

Conversely, let $B$ be a minimal relative generating set of $\mathcal{O P}(X, Y)$ modulo $\mathcal{O}(X, Y)$. By Lemma 3, there is a set $\bar{B} \subseteq B$ such that $\mathcal{P}_{m}=$ $\{\operatorname{ker} \beta: \beta \in \bar{B}\}$ and $|\bar{B}|=\left|\mathcal{P}_{m}\right|$. Since $\mathcal{O P}(X, Y)=\langle\mathcal{O}(X, Y), B\rangle$, there are $\beta_{1}, \ldots, \beta_{k} \in \mathcal{O}(X, Y) \cup B$ such that $\eta=\beta_{1} \cdots \beta_{k}$. Without loss of generality, we can assume that there is not $\gamma \in\left\{\beta_{i}: 1 \leq i \leq k, \operatorname{ker} \beta_{i} \in \mathcal{Q}_{m}\right\}=: \widehat{B}$ such that $\eta$ is a product of transformations in $\bar{B} \cup(\widehat{B} \backslash\{\gamma\})$. In the first part of the proof, we have shown that $\bar{B} \cup \widehat{B}$ is a relative generating set of $\mathcal{O P}(X, Y)$ modulo $\mathcal{O}(X, Y)$. Because of the minimality of $B$, we have $B=\bar{B} \cup \widehat{B}$, where $\{\operatorname{ker} \beta: \beta \in B \backslash \widehat{B}\} \supseteq \mathcal{P}_{m},|B \backslash \widehat{B}|=|\bar{B}|=\left|\mathcal{P}_{m}\right|$ and $\left.\eta\right|_{Y} \in\left\langle\left.\beta\right|_{Y}: \beta \in B\right\rangle$ but $\left.\eta\right|_{Y} \notin\left\langle\left.\beta\right|_{Y}: \beta \in B \backslash\{\gamma\}\right\rangle$ for any $\gamma \in \widehat{B}$.

In particular, for the relative generating sets of minimal size we have 
Remark $7 B \subseteq \mathcal{O P}(X, Y)$ is a relative generating set of $\mathcal{O P}(X, Y)$ modulo $\mathcal{O}(X, Y)$ of minimal size if and only if $|\widetilde{B}|=1$ if $1, n \in Y$ and $\widetilde{B}=\emptyset$, otherwise.

\section{The relative rank of $\mathcal{T}(X, Y)$ modulo $\mathcal{O P}(X, Y)$}

In this section, we determine the relative rank of $\mathcal{T}(X, Y)$ modulo $\mathcal{O P}(X, Y)$ and characterize all minimal relative generating sets of $\mathcal{T}(X, Y)$ modulo $\mathcal{O P}(X, Y)$. Since $\mathcal{O}(X, Y) \leq \mathcal{O P}(X, Y)$, we see immediately that $\operatorname{rank}(\mathcal{T}(X, Y)$ : $\mathcal{O P}(X, Y)) \leq S(n, m)-\left(\begin{array}{c}n-1 \\ m-1\end{array}\right)+1$. First, we state a sufficient condition for a set $B \subseteq \mathcal{T}(X, Y)$ to be a relative generating set of $\mathcal{T}(X, Y)$ modulo $\mathcal{O P}(X, Y)$.

Lemma 8 Let $B \subseteq \mathcal{T}(X, Y)$. If $\mathcal{R}_{m} \subseteq\{\operatorname{ker} \beta: \beta \in B\}$ and $\mathcal{S}(Y) \subseteq$ $\left\langle\left\{\left.\beta\right|_{Y}: \beta \in B\right\},\left.\eta\right|_{Y}\right\rangle$ then $\langle\mathcal{O P}(X, Y), B\rangle=\mathcal{T}(X, Y)$.

Proof. Let $\gamma \in \mathcal{T}(X, Y)$ with $\operatorname{rank} \gamma=k \leq m$. We will consider two cases.

Case 1. Suppose that $\operatorname{ker} \gamma \in \mathcal{R}_{k}$. Then $\operatorname{ker} \gamma$ contains a non-convex set which cannot be decomposed into two convex sets, which contain 1 and $n$, respectively. Since $k \leq m$, we can divide the partition ker $\gamma$ into a partition $P \in \mathcal{R}_{m}$ such that $P$ contains a non-convex set which cannot be decomposed into two convex sets, which contain 1 and $n$, respectively (if $k=m$ then we put $P=\operatorname{ker} \gamma$ ). Since $\mathcal{R}_{m} \subseteq\{\operatorname{ker} \beta: \beta \in B\}$, there is $\lambda \in B$ with ker $\lambda=P$. It is clear that $X \lambda=Y$.

Further, let $X \gamma=\left\{y_{1}<y_{2}<\cdots<y_{k}\right\}$ and define the sets

$$
A_{i}=\left\{x \in Y: x \lambda^{-1} \subseteq y_{i} \gamma^{-1}\right\}
$$

for $i=1, \ldots, k$. It is clear that $\left\{A_{1}, A_{2}, \ldots, A_{k}\right\}$ is a partition of $Y$. Moreover, let $\left\{C_{1}<C_{2}<\cdots<C_{k}\right\} \in \mathcal{Q}_{k}$ be a partition of $X$ such that $\left|C_{i} \cap Y\right|=\left|A_{i}\right|$ for all $i=1, \ldots, k$. Let $A_{i}=\left\{a_{i_{1}}<a_{i_{2}}<\cdots<a_{i_{i}}\right\}$ and $C_{i} \cap Y=\left\{c_{i_{1}}<c_{i_{2}}<\cdots<c_{i_{i}}\right\}$ with $t_{i} \in\{1, \ldots, m\}$ for $i \in\{1, \ldots, k\}$. We define a bijection

$$
\sigma: \bigcup_{i=1}^{k} A_{i}=Y \longrightarrow \bigcup_{i=1}^{k}\left(C_{i} \cap Y\right)=Y
$$

on $Y$ with $a_{i_{l}} \sigma=c_{i_{l}}$, for $l=1, \ldots, t_{i}$ and $i=1, \ldots, k$. Since $\sigma \in \mathcal{S}(Y)$ and $\mathcal{S}(Y) \subseteq\left\langle\left\{\left.\beta\right|_{Y}: \beta \in B\right\},\left.\eta\right|_{Y}\right\rangle$ there is $\mu \in\langle B, \eta\rangle$ with $\left.\mu\right|_{Y}=\sigma$. 
Finally, we define a transformation $\nu \in \mathcal{O}(X, Y) \subseteq \mathcal{O P}(X, Y)$ with $\operatorname{ker} \nu=$ $\left\{C_{1}<C_{2}<\cdots<C_{k}\right\}$ and $x \nu=y_{i}$ for all $x \in C_{i}$ and $i=1, \ldots, k$.

Therefore, we have $\lambda, \mu, \nu \in\langle\mathcal{O P}(X, Y), B\rangle$ and it remains to show that $\gamma=\lambda \mu \nu$, i.e. $\gamma \in\langle\mathcal{O P}(X, Y), B\rangle$. Let $x \in X$. Then $x \gamma=y_{i}$ for some $i \in\{1, \ldots, k\}$ and we have

$$
x \gamma=y_{i} \Rightarrow x \lambda=z \in A_{i} \Rightarrow z \mu=u \in C_{i} \cap Y \Rightarrow u \nu=y_{i} .
$$

Hence, $x \gamma=y_{i}=x(\lambda \mu \nu)$ and we conclude $\gamma=\lambda \mu \nu$.

Case 2. Suppose that $\operatorname{ker} \gamma \notin \mathcal{R}_{k}$, i.e. $\operatorname{ker} \gamma \in \mathcal{Q}_{k} \cup \mathcal{P}_{k}$ and there is $\rho_{1} \in \mathcal{O P}(X, Y)$ with $\operatorname{ker} \rho_{1}=\operatorname{ker} \gamma$. Further, there is a partition $P=\left\{D_{y}\right.$ : $\left.y \in X \rho_{1}\right\} \in \mathcal{R}_{k}$ such that $y \in D_{y}$, for all $y \in X \rho_{1}$. Then we define a transformation $\rho_{2}: X \rightarrow X \gamma$ with $\operatorname{ker} \rho_{2}=P$ and $\left\{x \rho_{2}\right\}=y \rho_{1}^{-1} \gamma$ for all $x \in D_{y}$ and $y \in X \rho_{1}$. Since $\operatorname{ker} \rho_{1}=\operatorname{ker} \gamma$, the transformation $\rho_{2}$ is well defined and we have $\gamma=\rho_{1} \rho_{2}$. Moreover, $\rho_{2} \in\langle\mathcal{O P}(X, Y), B\rangle$ by Case 1 (since ker $\rho_{2} \in \mathcal{R}_{k}$ ) and thus $\gamma=\rho_{1} \rho_{2} \in\langle\mathcal{O P}(X, Y), B\rangle$.

Lemma $9\left\langle\left.\eta\right|_{Y}\right\rangle=\left\langle\left\{\left.\beta\right|_{Y}: \beta \in \mathcal{O P}(X, Y)\right\}\right\rangle \cap \mathcal{S}(Y)$.

Proof. The inclusion $\left\langle\left.\eta\right|_{Y}\right\rangle \subseteq\left\langle\left\{\left.\beta\right|_{Y}: \beta \in \mathcal{O P}(X, Y)\right\}\right\rangle \cap \mathcal{S}(Y)$ is obviously. Let now $\beta \in \mathcal{O P}(X, Y)$ with $\left.\beta\right|_{Y} \in \mathcal{S}(Y)$. Then there is $k \in\{1, \ldots, m\}$ such that

$$
\beta=\left(\begin{array}{cccccc}
A_{1} & \cdots & A_{m-k+1} & A_{m-k} & \cdots & A_{m} \\
a_{k} & \cdots & a_{m} & a_{1} & \cdots & a_{k-1}
\end{array}\right)
$$

with $\left\{A_{1}, A_{2}<\cdots<A_{m}\right\} \in \mathcal{P}_{m} \cup \mathcal{Q}_{m}$ and $a_{i} \in A_{i}$ for $i \in\{1, \ldots, m\}$ since $Y$ is a transversal of $\operatorname{ker} \beta$. Thus,

$$
\left.\beta\right|_{Y}=\left(\begin{array}{cccccc}
a_{1} & \cdots & a_{m-k+1} & a_{m-k} & \cdots & a_{m} \\
a_{k} & \cdots & a_{m} & a_{1} & \cdots & a_{k-1}
\end{array}\right)=\left(\left.\eta\right|_{Y}\right)^{m-k+1} \in\left\langle\left.\eta\right|_{Y}\right\rangle .
$$

This shows that $\left\langle\left\{\left.\beta\right|_{Y}: \beta \in \mathcal{O P}(X, Y)\right\}\right\rangle \cap \mathcal{S}(Y) \subseteq\left\{\left(\left.\eta\right|_{Y}\right)^{p}: p \in \mathbb{N}\right\}=\left\langle\left.\eta\right|_{Y}\right\rangle$.

The following lemmas give us necessary conditions for a set $B \subseteq \mathcal{T}(X, Y)$ to be a relative generating set of $\mathcal{T}(X, Y)$ modulo $\mathcal{O P}(X, Y)$.

Lemma 10 Let $B \subseteq \mathcal{T}(X, Y) \backslash \mathcal{O P}(X, Y)$ with $\langle\mathcal{O P}(X, Y), B\rangle=\mathcal{T}(X, Y)$. Then $\mathcal{S}(Y) \subseteq\left\langle\left\{\left.\beta\right|_{Y}: \beta \in B\right\},\left.\eta\right|_{Y}\right\rangle$.

Proof. Let $\sigma \in \mathcal{S}(Y)$. We extend $\sigma$ to a transformation $\gamma: X \rightarrow Y$, i.e. $\left.\gamma\right|_{Y}=\sigma$. Hence, there are $\gamma_{1}, \ldots, \gamma_{k} \in \mathcal{O} \mathcal{P}(X, Y) \cup B$ (for a suitable natural 
number $k$ ) such that $\gamma=\gamma_{1} \cdots \gamma_{k}$. Since the image of any transformation in $\mathcal{T}(X, Y)$ belongs to $Y$, we have $\sigma=\left.\gamma\right|_{Y}=\left.\left.\gamma_{1}\right|_{Y} \cdots \gamma_{k}\right|_{Y}$. Moreover, from $\sigma \in \mathcal{S}(Y)$, we conclude $\left.\gamma_{i}\right|_{Y} \in \mathcal{S}(Y)$ for $1 \leq i \leq k$. Let $\gamma_{i} \in \mathcal{O P}(X, Y)$ for some $i \in\{1, \ldots, k\}$. Then by Lemma 9

$$
\left.\gamma_{i}\right|_{Y}=\left(\begin{array}{cccccc}
a_{1} & \cdots & a_{t} & a_{t+1} & \cdots & a_{m} \\
a_{m-t+1} & \cdots & a_{m} & a_{1} & \cdots & a_{m-t}
\end{array}\right) \in\left\langle\left.\eta\right|_{Y}\right\rangle
$$

for a suitable natural number $t$. This shows $\sigma \in\left\langle\left\{\left.\beta\right|_{Y}: \beta \in B\right\},\left.\eta\right|_{Y}\right\rangle$.

Lemma 11 Let $B \subseteq \mathcal{T}(X, Y) \backslash \mathcal{O P}(X, Y)$ with $\langle\mathcal{O P}(X, Y), B\rangle=\mathcal{T}(X, Y)$. Then $\mathcal{R}_{m} \subseteq\{\operatorname{ker} \beta: \beta \in B\}$.

Proof. Assume that there is $P \in \mathcal{R}_{m}$ with $P \notin\{\operatorname{ker} \beta: \beta \in B\}$. Let $\gamma \in \mathcal{T}(X, Y)$ with $\operatorname{ker} \gamma=P$. Then there are $\theta_{1} \in \mathcal{O P}(X, Y) \cup B$ and $\theta_{2} \in \mathcal{T}(X, Y)$ such that $\gamma=\theta_{1} \theta_{2}$. Since $\operatorname{rank} \gamma=m$, we obtain $\operatorname{ker} \gamma=$ $\operatorname{ker} \theta_{1}=P$. Thus, $\theta_{1} \notin B$, i.e. $\theta_{1} \in \mathcal{O P}(X, Y)$ and $\operatorname{ker} \theta_{1} \in \mathcal{Q}_{m} \cup \mathcal{P}_{m}$, contradicts $\operatorname{ker} \theta_{1}=P \in \mathcal{R}_{m}$.

Lemma 11] shows that $\operatorname{rank}(\mathcal{T}(X, Y): \mathcal{O P}(X, Y)) \geq\left|\mathcal{R}_{m}\right|$. We will verify the equality.

Lemma $12\left|\mathcal{R}_{m}\right|=S(m, n)-\left(\begin{array}{c}n \\ m\end{array}\right)$.

Proof. The cardinality of the set $\mathcal{D}_{m}:=\mathcal{R}_{m} \cup \mathcal{P}_{m}$ was determined in [16]. The authors show that $\left|\mathcal{D}_{m}\right|=S(m, n)-\left(\begin{array}{c}n-1 \\ m-1\end{array}\right)$. Because of $\mathcal{R}_{m} \cap \mathcal{P}_{m}=$ $\emptyset$, we obtain $\mathcal{R}_{m}=\mathcal{D}_{m} \backslash \mathcal{P}_{m}$. Since $\left|\mathcal{P}_{m}\right|=\left(\begin{array}{c}n-1 \\ m\end{array}\right)$ (see Remark 4) it follows $\left|\mathcal{R}_{m}\right|=\left|\mathcal{D}_{m}\right|-\left|\mathcal{P}_{m}\right|=S(m, n)-\left(\begin{array}{c}n-1 \\ m-1\end{array}\right)-\left(\begin{array}{c}n-1 \\ m\end{array}\right)=$ $S(m, n)-\left(\begin{array}{c}n \\ m\end{array}\right)$.

Finally, we can state the relative rank of $\mathcal{T}(X, Y)$ modulo $\mathcal{O P}(X, Y)$.

Theorem $13 \operatorname{rank}(\mathcal{T}(X, Y): \mathcal{O} \mathcal{P}(X, Y))=S(m, n)-\left(\begin{array}{c}n \\ m\end{array}\right)$.

Proof. If $m=1$ then $\mathcal{T}(X, Y)=\mathcal{O P}(X, Y)$, i.e. $\operatorname{rank}(\mathcal{T}(X, Y): \mathcal{O P}(X, Y))=$ 0 . On the other hand, we have $S(1, n)=n=\left(\begin{array}{c}n \\ 1\end{array}\right)$. Suppose now that 
$n \geq 2$. By Lemmas 11 and 12, we obtain $\operatorname{rank}(\mathcal{T}(X, Y): \mathcal{O P}(X, Y)) \geq$ $\left|\mathcal{R}_{m}\right|=S(m, n)-\left(\begin{array}{c}n \\ m\end{array}\right)$. In order to prove the equality, we have to find a relative generating set $B$ of $\mathcal{T}(X, Y)$ modulo $\mathcal{O P}(X, Y)$ with $|B|=\left|\mathcal{R}_{m}\right|$. We observe that for each $P \in \mathcal{R}_{m}$, there is $\beta_{P} \in \mathcal{T}(X, Y)$ with $\operatorname{ker} \beta_{P}=P$, which will be fixed. Let $\mathcal{B}:=\left\{\beta_{P}: P \in \mathcal{R}_{m}\right\}$. If $m=2$ then $\mathcal{R}_{m}=\emptyset$ and $\mathcal{S}(Y)=\left\{\left.\eta\right|_{Y},\left(\left.\eta\right|_{Y}\right)^{2}\right\}=\left\langle\left.\eta\right|_{Y}\right\rangle$, obviously. If $m \geq 3$ then without loss of generality we can assume that there is $P^{\prime} \in \mathcal{R}_{m}$ such that $Y$ is a transversal of $\operatorname{ker} \beta_{P^{\prime}}$ and $\left.\beta_{P^{\prime}}\right|_{Y}=\left(\begin{array}{ccccc}a_{1} & a_{2} & a_{3} & \cdots & a_{m} \\ a_{2} & a_{1} & a_{3} & \cdots & a_{m}\end{array}\right)$. It is well known that $\mathcal{S}(Y)=\left\langle\left.\beta_{P^{\prime}}\right|_{Y},\left.\eta\right|_{Y}\right\rangle$. Hence, $\mathcal{B}$ is a relative generating set of $\mathcal{T}(X, Y) \bmod -$ ulo $\mathcal{O P}(X, Y)$ by Lemma 8 . Since $|\mathcal{B}|=\left|\mathcal{R}_{m}\right|$, we obtain the required result.

Now we will characterize the minimal relative generating sets of $\mathcal{T}(X, Y)$ modulo $\mathcal{O P}(X, Y)$. The minimal relative generating sets do not coincide with the relative generating sets of $\operatorname{size} \operatorname{rank}(\mathcal{T}(X, Y): \mathcal{O P}(X, Y))$.

Theorem 14 Let $B \subseteq \mathcal{T}(X, Y)$. Then $B$ is a minimal relative generating set of $\mathcal{T}(X, Y)$ modulo $\mathcal{O P}(X, Y)$ if and only if there is a set $\widetilde{B} \subseteq B$ such that the following three statements are satisfied:

(i) $\mathcal{R}_{m} \subseteq\{\operatorname{ker} \beta: \beta \in B \backslash \widetilde{B}\}$,

(ii) $|B \backslash \widetilde{B}|=\left|\mathcal{R}_{m}\right|$,

(iii) $\mathcal{S}(Y) \subseteq\left\langle\left\{\left.\beta\right|_{Y}: \beta \in B\right\},\left.\eta\right|_{Y}\right\rangle$ but $\mathcal{S}(Y) \nsubseteq\left\langle\left\{\left.\beta\right|_{Y}: \beta \in B \backslash\{\gamma\}\right\},\left.\eta\right|_{Y}\right\rangle$ for any $\gamma \in B$ with $\operatorname{ker} \gamma \in\{\operatorname{ker} \beta: \beta \in \widetilde{B}\}$.

Proof. Suppose that the conditions $(i)-(i i i)$ are satisfied. Then by Lemma 8 we have $\langle\mathcal{O P}(X, Y), B\rangle=\mathcal{T}(X, Y)$. It remains to show that $B$ is minimal. Assume that there is $\gamma \in B$ such that $\langle\mathcal{O P}(X, Y), B \backslash\{\gamma\}\rangle=\mathcal{T}(X, Y)$. Note that $\left.\alpha \beta\right|_{Y}=\left.\left.\alpha\right|_{Y} \beta\right|_{Y}$ for all $\alpha, \beta \in \mathcal{T}(X, Y)$. Hence, we can conclude that $\mathcal{S}(Y) \subseteq\left\langle\left\{\left.\beta\right|_{Y}: \beta \in \mathcal{T}(X, Y)\right\}\right\rangle \subseteq\left\langle\left\{\left.\beta\right|_{Y}: \beta \in \mathcal{O P}(X, Y) \cup(B \backslash\{\gamma\})\right\}\right\rangle=$ $\left\langle\left\{\left.\beta\right|_{Y}: \beta \in B \backslash\{\gamma\}\right\},\left.\eta\right|_{Y}\right\rangle$ by Lemma 9. Hence, $\operatorname{cor} \gamma \notin\{\operatorname{ker} \beta: \beta \in \widetilde{B}\}$ by (iii). This implies that $\gamma \in B \backslash \widetilde{B}$ and $|(B \backslash \widetilde{B}) \backslash\{\gamma\}|<\left|\mathcal{R}_{m}\right|$ by $($ ii $)$, i.e. $\mathcal{R}_{m} \nsubseteq\{\operatorname{ker} \beta: \beta \in(B \backslash \widetilde{B}) \backslash\{\gamma\}\}$. Since $\operatorname{ker} \gamma \notin\{\operatorname{ker} \beta: \beta \in \widetilde{B}\}$, we have $\mathcal{R}_{m} \nsubseteq\{\operatorname{ker} \beta: \beta \in(B \backslash\{\gamma\})\}$ and by Lemma 11, we obtain that $\langle\mathcal{O P}(X, Y), B \backslash\{\gamma\}\rangle \neq \mathcal{T}(X, Y)$, a contradiction. This shows that $B$ is a minimal relative generating set of $\mathcal{T}(X, Y)$ modulo $\mathcal{O P}(X, Y)$.

Conversely, let $B$ be a minimal relative generating set of $\mathcal{T}(X, Y)$ modulo $\mathcal{O P}(X, Y)$. We have $\mathcal{R}_{m} \subseteq\{\operatorname{ker} \beta: \beta \in B\}$ and $\mathcal{S}(Y) \subseteq\left\langle\left\{\left.\beta\right|_{Y}: \beta \in B\right\},\left.\eta\right|_{Y}\right\rangle$ by Lemma 11 and Lemma [10, respectively. Then there exists a set $\mathbb{B} \subseteq B$ with $|B \backslash \widetilde{B}|=\left|\mathcal{R}_{m}\right|$ and $\mathcal{R}_{m} \subseteq\{\operatorname{ker} \beta: \beta \in(B \backslash \widetilde{B})\}$. For the set $\widetilde{B}$, 
the conditions $(i)$ and $(i i)$ are satisfied. Assume now that there is $\gamma \in B$ with $\operatorname{ker} \gamma \in\{\operatorname{ker} \beta: \beta \in \widetilde{B}\}$ such that $\mathcal{S}(Y) \subseteq\left\langle\left\{\left.\beta\right|_{Y}: \beta \in B \backslash\{\gamma\}\right\},\left.\eta\right|_{Y}\right\rangle$. Then because of $\mathcal{R}_{m} \subseteq\{\operatorname{ker} \beta: \beta \in(B \backslash\{\gamma\})\}$, the set $B \backslash\{\gamma)$ is a relative generating set of $\mathcal{T}(X, Y)$ modulo $\mathcal{O P}(X, Y)$ by Lemma 8 , This contradicts the minimality of $B$. Consequently, (iii) is satisfied.

In particular, for the relative generating sets of minimal size we have

Remark $15 B \subseteq \mathcal{T}(X, Y)$ is a relative generating set of $\mathcal{T}(X, Y)$ modulo $\mathcal{O P}(X, Y)$ of minimal size if and only if $\widetilde{B}=\emptyset$.

\section{References}

[1] Catarino P. M., Higgins P. M., The monoid of orientation-preserving mappings on a chain, Semigroup Forum, 58 (1999), 190-206.

[2] Dimitrova I., Fernandes V. H., Koppitz J., A note on generators of the endomorphism semigroup of an infinite countable chain, Journal of algebra and its applications, 16(2) (2017), 1750031.

[3] Dimitrova I., Koppitz J., Tinpun K., On the relative rank of the semigroup of orientation-preserving transformations with restricted range, Proceedings of the 47-th Spring Conference of the Union of Bulgarian Mathematicians, (2018), 109-114.

[4] Fernandes V. H., Honyam P., Quinteiro T. M., Singha B., On semigroups of endomorphisms of a chain with restricted range, Semigroup Forum, 89 (2014), 77-104.

[5] Fernandes V. H., Honyam P., Quinteiro T. M., Singha B., On semigroups of orientation-preserving transformations with restricted range, Comm. Algebra, 44 (2016), 253-264.

[6] Fernandes V. H., Sanwong J., On the rank of semigroups of transformations on a finite set with restricted range, Algebra Colloq., 21 (2014), $497-510$.

[7] Gomes G. M. S., Howie J. M., On the rank of certain semigroups of order-preserving transformations, Semigroup Forum, 51 (1992), 275282. 
[8] Gomes G. M. S., Howie J. M., On the ranks of certain finite semigroups of transformations, Math. Proc. Cambridge Philos. Soc., 101 (1987), $395-403$.

[9] Higgins P. M., Mitchell J. D., Ruškuc N., Generating the full transformation semigroup using order preserving mappings, Glasgow Math. J., 45 (2003), 557-566.

[10] Howie J. M., Fundamentals of semigroup theory, Oxford, Oxford University Press, 1995.

[11] Howie J. M., McFadden R. B., Idempotent rank in finite full transformation semigroups, Proc. Royal Soc. Edinburgh, 114A (1990), 161-167.

[12] Howie J. M., Ruškuc N., Higgins P. M., On relative ranks of full transformation semigroups, Comm. Algebra, 26 (1998), 733-748.

[13] McAlister D., Semigroups generated by a group and an idempotent, Comm. in Algebra, 26 (1998), 515-547.

[14] Ruškuc N., On the rank of completely 0-simple semigroups, Math. Proc. Cambridge Philos. Soc., 116 (1994), 325-338.

[15] Symons J. S. V., Some results concerning a transformation semigroup, J. Austral. Math. Soc., 19 (1975), 413-425.

[16] Tinpun K., Koppitz J., Relative rank of the finite full transformation semigroup with restricted range, Acta Mathematica Universitatis Comenianae, 85(2) (2016), 347-356. 